\title{
Molecular Detection and Quantification Gene Expression of Efflux Pump Antibiotic Resistance Genes in Extensive Drug Resistance Pseudomonas Aeruginosa Isolated from Clinical Infection Patients in Al Diwaniyah City of Iraq
}

\author{
Hassan Hachim Naser ${ }^{1}$, Adnan H. Aubaid ${ }^{2}$ \\ ${ }^{1}$ Lecturer, Department of Medical Microbiology, College of Medicine, University of Al-Qadisiyah, Iraq, \\ ${ }^{2}$ Prof., Department of Medical Microbiology, College of Medicine, University of Al-Qadisiyah, Iraq
}

\begin{abstract}
The study was included investigated 16 extensive drug resistance pseudomonas aeruginosa isolates which previously isolated from sputum, burns, urine, and ear swab clinical patients samples. The reverse transcription Real Time PCR (RT-qPCR) was performed for molecular detection and quantification of gene expression levels for efflux pump antibiotic resistance gene (mexA, mexB, mexC, mexE, and mexY) and chromosomal lactamase $(\mathrm{AmpC})$ gene, as well as outer membrane prion protein (OprD) gene in extensive drug pseudomonas aeruginosa isolates. The relative expression analysis was done by used the $2-\Delta \Delta \mathrm{CT}$ (Livak) method, this method was used for normalization of expression levels (mRNA transcript levels) in target genes with suitable housekeeping gene. In this study 30S ribosomal protein S12 (rpsL) housekeeping gene was used in normalization of antibiotic resistance genes expression levels in extensive drug pseudomonas aeruginosa isolates. The efflux pump resistance gene expression were by RT-qPCR technique. The relative gene expression analysis were showed highest frequency of overexpression at $(100 \%, 100 \%, 87.5 \%$, $81.25 \%, 56.25 \%$ ) for (mexB, mexE, mexA,mexC, and mexY) with fold change ranged (2.16- 184.82) fold change compared to wild type PA control isolate. The relative gene expression analysis of chromosomal lactamase AmpC gene was showed highest frequency of overexpression $(100 \%)$ in XDR isolates with expression levels ranged (6.77- 73.1) fold change compared to wild type PA control isolate. Whereas, the relative gene expression analysis of OprD gene was showed (68.75\%) frequency of down expression in XDR Pseudomonas aeruginosa isolates with expression levels ranged (0.01-0.16) fold change compared to wild type PA control isolate. In conclusion, The XDR Pseudomonas aeruginosa isolates can ability to over expression production of inducible chromosomal lactamase AmpC gene, efflux pumps protein genes, and low expression outer membrane prion protein $(\mathrm{OprD})$ gene as important antimicrobial mechanism against Cephalosporins and Carbapenems antibiotic classes.
\end{abstract}

Keywords: Pseudomonas aeruginosa, XDR, efflux pumps genes, gene expression.

\section{Introduction}

The development of XDR Pseudomonas aeruginosa has become a genuine overall clinical issue. The decrease

\footnotetext{
Corresponding Author:

Hassan Hachim Naser

Lecturer, Department of Medical Microbiology, College of Medicine, University of Al-Qadisiyah, Iraq e-mail: hassan.naser@qu.edu.iq
}

of medication amassing in the cytoplasm is likewise a significant system to oppose anti-microbials. There are two different ways to accomplish the reason for lessening drug collection: one is through layer impermeability, and the other is by film related siphons ${ }^{1}$. The overexpression of efflux siphons was accounted for to add to multidrug opposition in P. aeruginosa ${ }^{2}$. Most Gram-negative microorganisms have the characteristics for efflux siphons having a spot with the restriction nodulation division family and a couple of homologous hindrance nodulation cell division-type siphons are delegate in $\mathrm{P}$. 
aeruginosa with the ultimate objective that MexAB-OprM (coding quality: mexA, mexB and oprM), MexCD-Opr $\mathrm{J}$ (mexC, mexD and opr J), MexEF-OprN (mexE, mexF and oprN), MexXY-OprA (mexX, mexY and oprA) and some $\mathrm{P}$. aeruginosa strains lost oprA quality ${ }^{3}$. MexABOprM adds to counter-agent poison assurance from $\beta$-lactams, for instance, cephalosporines or penicillins, macrolides, chloramphenicol, anti-infection prescription and fluoroquinolones MexCD-Opr $\mathrm{J}$ adds to antidote poison insurance from macrolides, anti-toxin drugs, fluoroquinolones and a couple $\beta$-lactams including cefepime. MexEF-OprN adds to hostile to microbial security from fluoroquinolones, chloramphenicol and trimethoprim. MexXY-(OprA) adds to hostile to contamination insurance from aminoglycosides, cefepime, ciprofloxacin and levofloxacin ${ }^{4,5}$. Mutational changes can cause diminished counter-agent poison take-up, alterations of against microbial targets, and overexpression of efflux siphons and hostile to contamination inactivating synthetics; all of which license minuscule living beings to make due inside seeing antimicrobial particles ${ }^{6}$. For instance, an investigation by ${ }^{7}$ showed that inactivation of the DNA oxidative fix framework expands change frequencies in P. aeruginosa prompting upgraded $\beta$-lactamase creation and overexpression of the MexCD-Opr J efflux siphon. Unconstrained changes can influence the articulation or capacity of a particular porin, consequently diminishing bacterial layer penetrability and expanding anti-toxin opposition ${ }^{8}$. Our examination was meant to measurement quality articulation of efflux siphon anti-toxin opposition qualities by turn around record Constant PCR (RTqPCR) in broad medication obstruction pseudomonas aeruginosa segregated from Clinical disease patients in Al Diwaniyah city of Iraq.

\section{Material and Method}

Bacterial Isolates: A 16 XDR Pseudomonas aeruginosa isolates were provided from Microbiology laboratory in college of Medicine. These XDR isolates were previously isolated from different official hospitals includes Al-Diwaniyah teaching hospital, Diwaniyah burn center hospital, Diwaniyah Chest center hospitals, as well as from many private medical laboratory in AlDiwaniyah city.

Quantitative Reverse Transcription Real-Time PCR: The quantitative Real-Time PCR technique was performed for quantification detection and gene expression analysis of antibiotics resistance efflux pumps genes and normalized by housekeeping (rpsL) gene in extensive multidrug resistance Pseudomonas aeruginosa isolates was carried out according to method described by ${ }^{2}$ and include the following steps:

Total RNA extraction: The XDR Pseudomonas aeruginosa bacterial isolates were inoculated on Luria Bertani broth and incubated at $37^{\circ} \mathrm{C}$ into reach bacterial cells (OD600:0.8-1.0), the bacterial cells were harvested by centrifuge at $13000 \mathrm{rpm}$ for $1 \mathrm{~min}$ then, supernatant removed. Total RNA was extracted by using (easyBLUETM Total RNA Extraction Kit) and done according to company instructions.

Estimation of extracted total RNA: The extracted total RNA was checked by using Nanodrop (Thermo Scientific Nano Drop Lite UV Visible Spectrophotometer. USA) that measured RNA concentration $(\mathrm{ng} / \mu \mathrm{L})$ and checked the RNA purity at absorbance $(260 / 280 \mathrm{~nm})$.

DNase I treatment: The extracted RNA were treated with DNase I enzyme to remove the trace amounts of genomic DNA from the eluted total RNA by using samples (DNase I enzyme kit) and done according to method described by Promega company, USA instructions as following table (1).

\section{Table (1): DNase I treatment mix preparation}

\begin{tabular}{|l|c|}
\hline Mix & Volume \\
\hline Total RNA $1 \mu \mathrm{g}$ & $10 \mu \mathrm{L}$ \\
\hline DNase I enzyme & $1 \mu \mathrm{L}$ \\
\hline 10X buffer & $4 \mu \mathrm{L}$ \\
\hline DEPC water & $5 \mu \mathrm{L}$ \\
\hline Total & $\mathbf{1 0 \mu \mathrm { L }}$ \\
\hline
\end{tabular}

From that point onward, The blend was brooded at $37 \mathrm{C}^{\circ}$ for 30 minutes. At that point, $1 \mu \mathrm{l}$ stop response was included and hatched at $65 \mathrm{C}^{\circ}$ for 10 minutes for inactivation of DNase catalyst activity.

cDNA combination: The DNase treated complete separated RNA tests were utilized in cDNA combination step from mRNA records by utilizing (AccuPower ${ }^{\circledR}$ RocketScriptTM RT PreMix) and this pack was finished by organization directions as following table (2).

Table (2): cDNA master mix preparation

\begin{tabular}{|l|c|}
\hline RT mix & Volume \\
\hline Total RNA $100 \mu \mathrm{g}$ & $10 \mu \mathrm{L}$ \\
\hline Random Hexamer primer $(50 \mathrm{pmol})$ & $1 \mu \mathrm{L}$ \\
\hline DEPC water & $9 \mu \mathrm{L}$ \\
\hline Total & $\mathbf{1 0 u l}$ \\
\hline
\end{tabular}


Table (3): cDNA synthesis thermocycler conditions protocol

\begin{tabular}{|l|c|c|}
\hline Step & Temperature & Time \\
\hline cDNA synthesis (RT step) & $42^{\circ} \mathrm{C}$ & 1 hour \\
\hline Heat inactivation & $95^{\circ} \mathrm{C}$ & 5 minutes \\
\hline
\end{tabular}

After that, these RT mix components that mentioned in table above placed in AccuPower ${ }^{\circledR}$ RocketScriptTM RT PreMix kit strip tubes that containing all other components which needed to cDNA synthesis such as (Reverse Transcriptase, $5 \times$ Reaction Buffer, DTT, dNTP, and RNase Inhibitor). Then, all the strip tubes transferred into Exispin vortex centrifuge at 3000rpm for 3 minutes, and then incubated in Thermocycler (BioRad-USA) as following thermocycler conditions protocol as showed in table (3)

Real-Time PCR (qPCR) master mix preparation: qPCR master mix was prepared by using (RealMODTM Green SF 2X qPCR mix Kit) based on SYBER green dye amplification in Real-Time PCR system and the qPCR master mix was prepared as following:

Real-Time PCR primers: Real Time PCR primers were designed in this study using NCBI-Genbank database and primer3 plus online. These primers were provided by Macrogencompany from Korea as following table (4).

Table (4): Real Time PCR primers for efflux pumps gene expression with their nucleotide sequence and product size.

\begin{tabular}{|c|c|c|c|}
\hline Primer & & Sequence (5'-3') & Product Size \\
\hline \multirow{2}{*}{ MexA } & $\mathrm{F}$ & TCGAATTCTCCGAGGTTTCCG & \multirow{2}{*}{$142 \mathrm{bp}$} \\
\hline & $\mathrm{R}$ & AGGATGGCCTTCTGCTTGAC & \\
\hline \multirow{2}{*}{ MexC } & $\mathrm{F}$ & TGGCTGATTTGCGTGCAATAG & \multirow{2}{*}{$115 \mathrm{bp}$} \\
\hline & $\mathrm{R}$ & TCCACCGGCAACACCATTTC & \\
\hline \multirow{2}{*}{ MexE } & $\mathrm{F}$ & TGGAACAGTCATCCCACTTCTC & \multirow{2}{*}{$145 \mathrm{bp}$} \\
\hline & $\mathrm{R}$ & AGCGGTTGTTCGATGACTTC & \\
\hline \multirow{2}{*}{ MexY } & $\mathrm{F}$ & GTCGTTGAAGAAGCCGTTTACC & \multirow{2}{*}{$122 \mathrm{bp}$} \\
\hline & $\mathrm{R}$ & GTGCAAACAAGCGCAACAAG & \\
\hline \multirow{2}{*}{ MexB } & $\mathrm{F}$ & CGTCAAGCAATTGCCGAAAG & \multirow{2}{*}{$150 \mathrm{bp}$} \\
\hline & $\mathrm{R}$ & ATCGACCAGCTTTCGTACAGG & \\
\hline \multirow{2}{*}{ AmpC } & $\mathrm{F}$ & ATGCGCGATACCAGATTCCC & \multirow{2}{*}{$146 \mathrm{bp}$} \\
\hline & $\mathrm{R}$ & TTGGCCTTCATCACCGGTTG & \\
\hline \multirow{2}{*}{ OprD } & $\mathrm{F}$ & AAGAGCGCCGATTTCATTGG & \multirow{2}{*}{$125 \mathrm{bp}$} \\
\hline & $\mathrm{R}$ & AGTGGGATGGTGTAGTTGCTG & \\
\hline \multirow{2}{*}{ Housekeeping gene rpsL } & $\mathrm{F}$ & TATGCACCCGCGTATACACC & \multirow{2}{*}{$93 \mathrm{bp}$} \\
\hline & $\mathrm{R}$ & AAACCTCGAAACCGTTGGTC & \\
\hline
\end{tabular}

Standard Curve qPCR master mix: qPCR standard curve was performed to evaluate the qPCR efficiency by Real-Time PCR amplification and prepared by using DNA Copy number calculator, online to calculate $\left(1 \times 10^{\wedge} 8\right)$ DNA copy number Pseudomonas aeruginosa, then used for prepared 10 fold dilution as $\left(1 \times 10^{\wedge} 8,1 \times 10^{\wedge} 7,1 \times 10^{\wedge} 6,1 \times 10^{\wedge} 5,1 \times 10^{\wedge} 4,1 \times 10^{\wedge} 3\right.$, $\left.1 \times 10^{\wedge} 2,1 \times 10^{\wedge} 1\right)$ that used in $\mathrm{qPCR}$ master mix as following table (5).
Table (5): qPCR standard curve master mix protocol

\begin{tabular}{|l|c|}
\hline qPCR master mix & Volume \\
\hline $\begin{array}{l}\text { 10 fold dilution (10ng) } \\
\text { DNA template }\end{array}$ & $5 \mu \mathrm{L}$ \\
\hline rpsL Forward primer(10pmol) & $1 \mu \mathrm{L}$ \\
\hline rpsL Reverse primer (10pmol) & $1 \mu \mathrm{L}$ \\
\hline qPCR Master Mix & $10 \mu \mathrm{L}$ \\
\hline Nuclease free water & $3 \mu \mathrm{L}$ \\
\hline Total & $\mathbf{2 0} \boldsymbol{\mu L}$ \\
\hline
\end{tabular}


Data analysis of qPCR: The data results of qPCR for target and housekeeping gene were used expression analysis (fold change) used (The $\Delta \mathrm{CT}$ Method Using a reference gene) that described by ${ }^{[9]}$ as following equation:

$$
\text { Ratio (reference/target })=2^{\mathrm{CT}(\text { reference })-\mathrm{CT}(\text { target })}
$$

Statistics Analysis: The statistical analysis was done by using LSD test, one way anova at $\mathrm{P}$ value $<0.05$ were performed by using GraphPad Prism 7 Statistics software.

\section{Results and Discussion}

The converse record Continuous PCR (RTqPCR) was performed for sub-atomic recognition and evaluation of quality articulation levels for efflux siphon anti-toxin obstruction quality (mexA, mexB, mexC, mexE, and mexY) and chromosomal lactamase (AmpC) quality, just as external layer prion protein (OprD) quality in broad medication pseudomonas aeruginosa separates. The relative articulation investigation was finished by utilized the 2- $\Delta \Delta \mathrm{CT}$ (Livak) strategy, this technique was utilized for standardization of articulation levels (mRNA record levels) in target qualities with appropriate housekeeping quality. In this investigation 30S ribosomal protein S12 (rpsL) housekeeping quality was utilized in standardization of anti-toxin obstruction qualities articulation levels in broad medication pseudomonas aeruginosa detaches as appeared in figure (3). The constant PCR productively was dictated by utilizing genomic DNA standard bend and the qPCR intensification plot results for three 10 overlay weakened DNA (1x102, 1x104, 1x106, and 1x108/duplicate number) with RT-PCR effectiveness (100\%) were appeared in figure (1) \& (2).

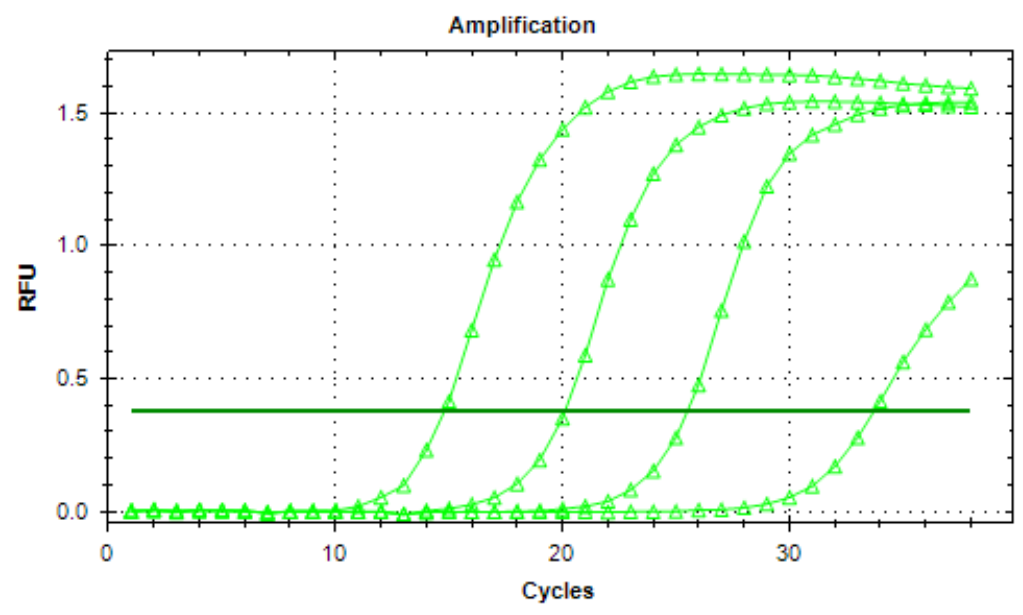

Figure (1): Real-Time PCR amplification plot of 10 fold diluted DNA template.

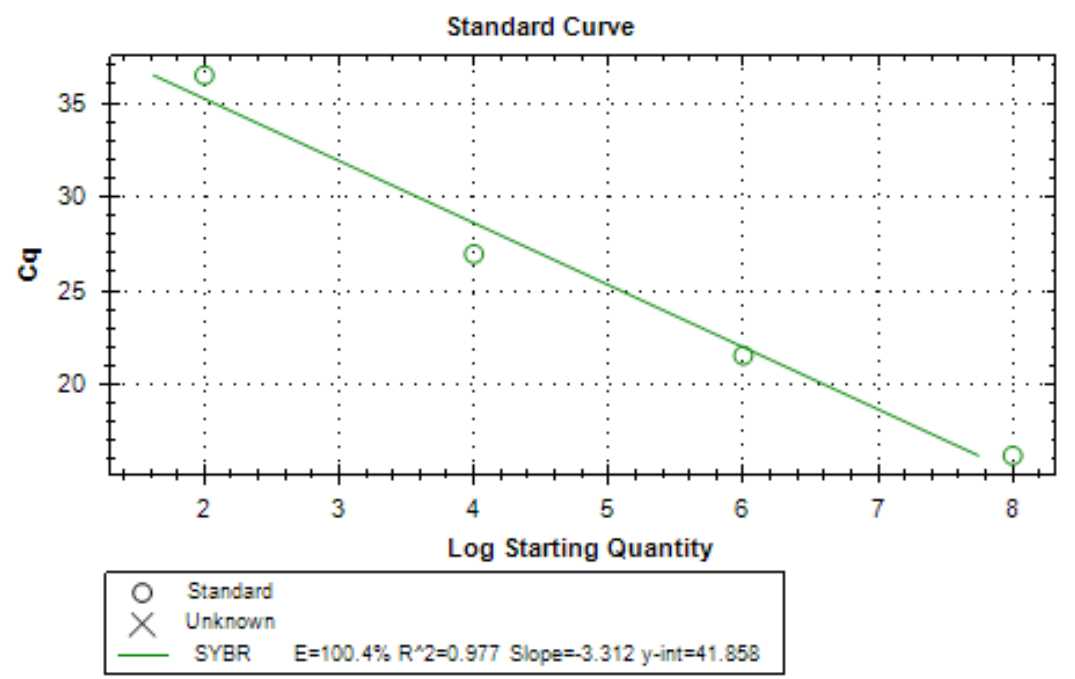

Figure (2): Ongoing PCR standard bend of 10 overlay weakened DNA format with qPCR proficiency (100\%). 


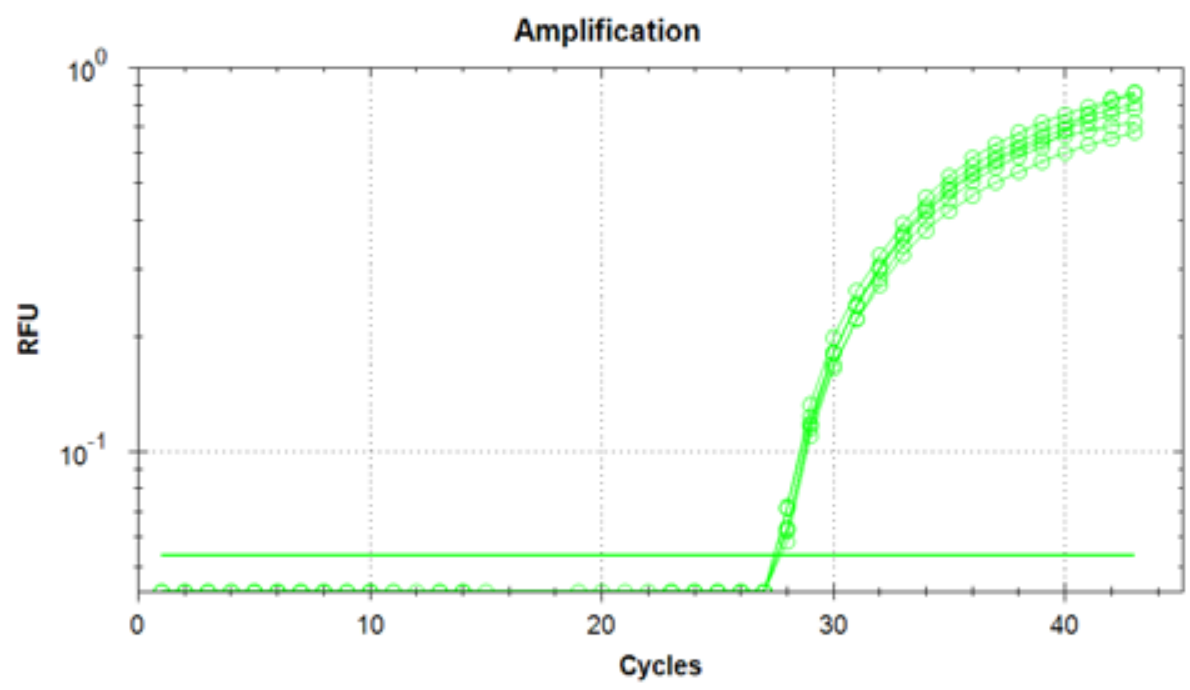

Figure (3): Constant PCR intensification plot of 30S ribosomal protein S12 (rpsL) housekeeping quality, that indicated no huge contrast in edge cycle numbers between broad medication pseudomonas aeruginosa disengages ran (26.63-53) CT number.

The relative quality articulation investigation were demonstrated most elevated recurrence of overexpression as appeared in table (6):

Table (6): Livak technique for Relative quality articulation investigation in XDR Pseudomonas aeruginosa disengages.

\begin{tabular}{|l|c|c|c|c|c|}
\hline Gene & qPCR detection (\%) & $\begin{array}{c}\text { Fold change } \\
\left(\mathbf{2}^{\wedge}-\Delta \Delta \mathbf{C T}\right)\end{array}$ & Over expression (\%) & Down expression (\%) & No Change (\%) \\
\hline AmpC & $100 \%$ & $6.77-73.1$ & $100 \%$ & $0 \%$ & $0 \%$ \\
\hline mexA & $87.50 \%$ & $2.16-121.10$ & $87.50 \%$ & $0 \%$ & $0 \%$ \\
\hline mexB & $100 \%$ & $15.24-148.06$ & $100 \%$ & $0 \%$ & $0 \%$ \\
\hline mexC & $81.25 \%$ & $2.79-184.82$ & $81.25 \%$ & $0 \%$ & $0 \%$ \\
\hline mexE & $100 \%$ & $16.11-123.65$ & $100 \%$ & $0 \%$ & $0 \%$ \\
\hline mexY & $56.25 \%$ & $3.97-20.11$ & $56.25 \%$ & $0 \%$ & 0 \\
\hline OprD & $68.75 \%$ & $0.01-0.16$ & $0 \%$ & & $0 \%$ \\
\hline
\end{tabular}

The mean of overlap change quality articulation investigation in of efflux siphon anti-infection obstruction qualities in XDR Pseudomonas aeruginosa disconnects as appeared in figure (4):.

\section{Relative gene expression (Fold change)}

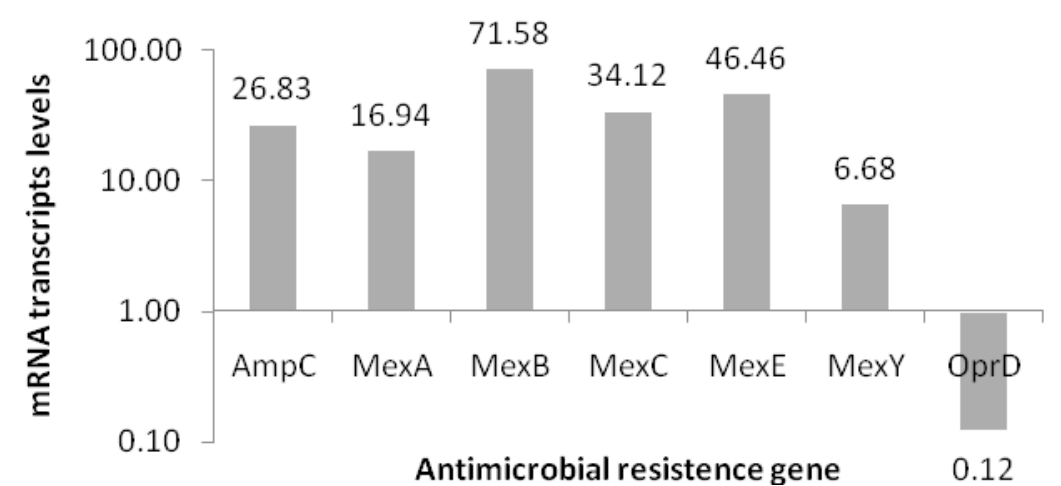

Figure (4): The mean of overlap change quality articulation investigation in of efflux siphon anti-toxin opposition qualities in XDR Pseudomonas aeruginosa detaches. 
The present study was showed that all XDR Pseudomonas aeruginosa isolates has significantly increased overexpression production of inducible chromosomal lactamase AmpC gene expression at $(100 \%)$ and their relative expression ratio ranged (6.7773.1) fold change compared to wild type PA control. This finding explains that overexpression of chromosomal $\mathrm{AmpC}(\mathrm{ampC})$ is mainly responsible for upregulation to resistance mechanism to penicillin and cephalosporinase $\beta$-lactams class as showed in our results for antibiotic susceptible pattern that (XDR) Pseudomonas aeruginosa isolates are resistance to cephalosporins. Study by ${ }^{[10]}$ who finding that mutation-dependent overproduction of intrinsic $\beta$-lactamase $\mathrm{AmpC}$ is considered the main cause ofresistance of clinical strains of Pseudomonas aeruginosa to antipseudomonalpenicillins and cephalosporins and these finding consistent with our results that exhibiting a greater resistance to ceftazidime and Cefepime as well as Piperacillin antibiotics. Another study in France showed increased resistance to ceftazidime among the clinical isolates of $\mathrm{P}$. aeruginosa it primarily because of AmpC over-expression $^{[11]}$. The present study was showed the relative gene expression analysis were showed highest frequency of overexpression at $(100 \%, 100 \%, 87.5 \%$, $81.25 \%, 56.25 \%)$ for $(\operatorname{mexB}, \operatorname{mexE}, \operatorname{mexA}, \operatorname{mexC}$, and mexY) with fold change ranged (2.16-184.82) fold change compared to wild type PA control isolate and all the XDR isolates were produced overexpression at relatively variable expression levels, it may be resulting from single mutation in efflux pump antibiotic resistance genes that effect on expression regulatory mechanisms. This suggestion consistence with previously observed results in the $\mathrm{P}$. aeruginosa clinical isolates [12]. Who finding that overexpression of the two efflux systems may result from mutations affecting multiple regulatory genes. The expression of AmpC is significantly increased, $\mathrm{P}$. aeruginosa is resistant to almost all classes of $\beta$-lactams, except the carbapenems ${ }^{[13]}$. Finally, our study was concluded that Pseudomonas aeruginosa is important causes of nosocomial diseases and has high ability to acquired multidrugs resistance capacity especially in burn infection patients, XDR Pseudomonas aeruginosa isolates can ability to overexpression production of inducible chromosomal lactamase AmpC gene, efflux pumps protein genes, and low expression outer membrane prion protein (OprD) gene as important antimicrobial mechanism against Cephalosporins and Carbapenems antibiotic classes.
Acknowledgements: Thanks are due to the Department of Medical Microbiology, College of Medicine, University of Al-Qadisiyah Iraq.

Financial Disclosure: There is no financial disclosure.

Conflict of Interest: None to declare.

Ethical Clearance: All experimental protocols were approved under the College of Medicine and all experiments were carried out in accordance with approved guidelines.

\section{References}

1. W Wu, Y Jin, FBai, S Jin. Pseudomonas aeruginosa. Elsevier Ltd, 2014; 2-3.

2. B Pourakbari, SYaslianifard, SYaslianifard, SMahmoudi. Evaluation of efflux pumps gene expression in resistant Pseudomonas aeruginosa isolates in an Iranian referral hospital," Iran. J. Microbiol.2016; 8: 249-256.

3. D Choudhury. Premature termination of MexR leads to overexpression of MexAB-OprM efflux pump in Pseudomonas aeruginosa in a tertiary referral hospital in India," PLoS One.2016; 11: $1-12$

4. JL Dumas, C Van Delden, KPerron, T Köhler. Analysis of antibiotic resistance gene expression in Pseudomonas aeruginosa by quantitative real-timePCR," FEMS Microbiol. Lett.2006; 254: 217-225

5. DE Xavier, RC Pico, R Girardello, L Fehlberg AC. Gales, "Efflux pumps expression and its association with porin down-regulation and $\beta$-lactamase production among Pseudomonas aeruginosa causing bloodstream infections in Brazil," BMC Microbiol.2010; 10

6. JMunita, C Arias, A Unit, A De Santiago, "HHS Public Access Mechanisms of Antibiotic Resistance," HHS Public Access.2016; 4: 1-37

7. LF Mandsberg, O Ciofu, N Kirkby, L E Christiansen, HEPoulsen, NHøiby, “Antibiotic resistance in Pseudomonas aeruginosa strains with increased mutation frequency due to inactivation of the DNA oxidative repair system," Antimicrob. Agents Chemother.2009; 53: 2483-2491.

8. LFernández, R Hancock. Adaptive and mutational resistance: Role of porins and efflux pumps in drug 
resistance," Clin. Microbiol. Rev.2012; 25: 661681

9. KJ Livak, TD Schmittgen. Analysis of Relative Gene Expression Data Using Real- Time Quantitative PCR and the $2 \times \bigcirc \bigcirc \mathrm{C}$ T Method. 2001;408: 402-408

10. MBerrazeg. Mutations in $\beta$-lactamase AmpC increase resistance of Pseudomonas aeruginosa isolates to antipseudomonalcephalosporins," Antimicrob. Agents Chemother.2015; 59: 62486255.

11. C De Champs. "Prospective survey of $\beta$-lactamases produced by ceftazidime-resistant Pseudomonas aeruginosa isolated in a French hospital in 2000," Antimicrob. Agents Chemother. 2002; 46: 30313034.

12. CLlanes. Clinical Strains of Pseudomonas aeruginosa Overproducing MexAB-OprM and MexXY Efflux Pumps Simultaneously Clinical Strains of Pseudomonas aeruginosa Overproducing MexAB-OprM and MexXY Efflux Pumps Simultaneously," Antimicrob. Agents Chemother.2004; 48: 1797-1802.

13. PD Lister, DJ Wolter, D Hanson. Antibacterialresistant Pseudomonas aeruginosa: Clinical impact and complex regulation of chromosomally encoded resistance mechanisms," Clin. Microbiol. Rev. 2009; 22: 582-610. 